\title{
Identification and application of bacterial volatiles to attract a generalist aphid parasitoid: from laboratory to greenhouse assays
}

Running title: Parasitoid attraction by bacterial volatiles

Tim Goelen, ${ }^{\text {a }}$ József Vuts, ${ }^{\mathrm{b}}$ Islam S. Sobhy, ${ }^{\mathrm{a}, \mathrm{c}}$ Felix Wäckers, ${ }^{\text {dee }}$ John C. Caulfield, ${ }^{\mathrm{b}}$ Michael A. Birkett, ${ }^{b}$ Hans Rediers, ${ }^{a}$ Hans Jacquemyn ${ }^{\mathrm{f}}$ and Bart Lievens ${ }^{\mathrm{a}^{*}}$

${ }^{a}$ Laboratory for Process Microbial Ecology and Bioinspirational Management (PME\&BIM), Centre of Microbial and Plant Genetics (CMPG), Department of Microbial and Molecular Systems (M2S), KU Leuven, B-3001 Leuven, Belgium.

${ }^{b}$ Department of Biointeractions and Crop Protection, Rothamsted Research, Harpenden, AL5 2JQ, UK.

${ }^{c}$ Department of Plant Protection, Faculty of Agriculture, Suez Canal University, 41522 Ismailia, Egypt.

${ }^{d}$ Biobest, B-2260 Westerlo, Belgium.

${ }^{e}$ Lancaster Environment Centre, Lancaster University, LA1 4YQ Lancaster, UK. ${ }^{f_{L}}$ aboratory of Plant Conservation and Population Biology, Biology Department, KU Leuven, B-3001 Leuven, Belgium.

This article has been accepted for publication and undergone full peer review but has not been through the copyediting, typesetting, pagination and proofreading process which may lead to differences between this version and the Version of Record. Please cite this article as doi: $10.1002 / p s .6102$ 
*Correspondence to: B Lievens, Laboratory for Process Microbial Ecology and Bioinspirational Management (PME\&BIM), KU Leuven, Willem De Croylaan 46, B3001 Leuven, Belgium. E-mail: bart.lievens@kuleuven.be. 
ORCID IDs:

TIM GOELEN: Orcid ID: 0000-0002-0034-6197

JOZSEF VUTS: Orcid ID: 0000-0001-6240-0905

ISLAM S. SOBHY: Orcid ID: 0000-0003-4984-1823

FELIX WACKERS: Orcid ID: 0000-0002-9748-0615

JOHN C. CAULFIELD: Orcid ID: 0000-0003-1799-370X

MICHAEL A. BIRKETT: Orcid ID: 0000-0002-3302-2025

HANS REDIERS : Orcid ID: 0000-0001-8645-107X

HANS JACQUEMYN: Orcid ID: 0000-0001-9600-5794

BART LIEVENS: Orcid ID: 0000-0002-7698-6641 


\begin{abstract}
BACKGROUND: Recent studies have shown that microorganisms emit volatile compounds that affect insect behaviour. However, it remains largely unclear whether microbes can be exploited as a source of attractants to improve biological control of insect pests. In this study, we used a combination of coupled gas chromatographyelectroantennography (GC-EAG) and Y-tube olfactometer bioassays to identify attractive compounds in the volatile extracts of three bacterial strains that are associated with the habitat of the generalist aphid parasitoid Aphidius colemani, and to create mixtures of synthetic compounds to find attractive blends for A. colemani. Subsequently, the most attractive blend was evaluated in two-choice cage experiments under greenhouse conditions.
\end{abstract}

RESULTS: GC-EAG analysis revealed 20 compounds that were linked to behaviourally attractive bacterial strains. A mixture of two EAG-active compounds, styrene and benzaldehyde applied at a respective dose of $1 \mu \mathrm{g}$ and $10 \mathrm{ng}$, was more attractive than the single compounds or the culture medium of the bacteria in Y-tube olfactometer bioassays. Application of this synthetic mixture under greenhouse conditions resulted in significant attraction of the parasitoids, and outperformed application of the bacterial culture medium.

CONCLUSION: Compounds isolated from bacterial blends were capable of attracting parasitoids both in laboratory and greenhouse assays, indicating that microbial cultures are an effective source of insect attractants. This opens new opportunities to attract and retain natural enemies of pest species and to enhance biological pest control. 
Keywords: Aphidius colemani; Bacillus; electroantennogram; multitrophic interactions; natural enemy; VOCs 


\section{INTRODUCTION}

Biological control using natural enemies such as arthropod predators and parasitoids has become an important alternative method of pest management, ${ }^{1}$ but the efficacy of biological pest control can be hampered when naturally occurring enemies are not sufficiently abundant or effective. ${ }^{2}$ To increase the efficacy of biological control, naturally occurring parasitoids and predators are often complemented with the release of commercially reared natural enemies. ${ }^{1,3}$ While this temporarily increases the local density of natural enemies, a major challenge in biological pest control remains to attract and retain beneficial insects within the crop so that they maintain high population densities in the longer term and sufficiently reduce the local abundance of pests. ${ }^{3,4}$

Insect- and plant-derived semiochemicals can be manufactured and deployed to manipulate the behaviour of natural enemies. Examples include volatiles produced when plants are attacked by herbivores (herbivore-induced plant volatiles, HIPVs), and alarm, sex or aggregation pheromones of pests or natural enemies. ${ }^{5,6}$ These chemicals can be sprayed onto crops or deployed in dispensers at regular intervals in the cropping system. ${ }^{5}$ While most research in this field has focused on cues derived from plants and insects, ${ }^{4,7}$ there is mounting evidence that microorganisms emit volatile compounds (mVOCs, microbial volatile organic compounds) that also play a role in insect behaviour. ${ }^{8-11}$ In some cases, mVOCs attract insects by signalling the presence of appropriate resources such as food sources and oviposition sites, ${ }^{12-14}$ whereas others have been found to deter insects. ${ }^{15}$ For example, yeast volatiles, rather than fruit volatiles, have been shown to be responsible for the attraction of the fruit fly Drosophila melanogaster to food resources 
and oviposition sites. ${ }^{12}$ Moreover, fruit flies use mVOCs to select yeasts that best support their growth and survival. ${ }^{16}$ Yeast volatile emissions have also been shown to play a major role in the attraction of flower visiting insects in floral nectar ${ }^{14,17}$ and serve as an excellent learning cue to locate suitable nectar sources. ${ }^{18}$ Similarly, mVOCs emitted by bacteria colonizing aphid honeydew have been shown to be attractive to tending ants, thereby mediating ant-aphid interactions. ${ }^{19}$ Besides locating food sources, mVOCs can also be exploited by natural enemies to locate hosts or preys, and even stimulate oviposition. ${ }^{13}$

Despite an increased understanding of the role of microbial volatiles as insect semiochemicals, ${ }^{8,9,20}$ little is still known whether they can be exploited as a source of attractants of pest natural enemies. In most cases, insects respond to complex mixtures of volatile compounds in specific ratios. ${ }^{21,22}$ However, other studies have shown that insects may also respond to single compounds. ${ }^{23,24}$ Additionally, there are examples indicating that simplified blends of synthetic volatiles, representing only a limited set of the volatiles from a natural blend, can be as attractive as the natural blends. ${ }^{25,26}$ This suggests that, despite the rich plethora of volatiles that are generally available from natural resources, only a select number of compounds evoke a behavioural response in the insects. So far, identification of active microbial compounds affecting parasitoid foraging behaviour, or mixtures thereof, and study of their performance under field conditions remain largely unexplored. Such studies would allow to fully grasp the potential of microbial volatiles to develop new semiochemical-based strategies to improve biological pest control efficacy. 
In previous research using laboratory assays with Aphidius colemani Viereck (Hymenoptera: Braconidae), we showed that parasitic wasps respond to volatile blends emitted by bacteria isolated from the parasitoids' habitat, ranging from significant attraction over no response to significant repellence. ${ }^{27}$ Preliminary analyses of the volatile blends showed that bacteria that significantly attracted the parasitoids produced blends with significantly lower amounts of esters, organic acids, aromatics and cycloalkanes than repellent strains. ${ }^{27}$ In this study, we tested the behavioural and electrophysiological responses of $A$. colemani females to the volatile blends of three bacterial strains producing attractive mVOCs. Subsequently, five EAG-active compounds were selected and tested individually, as well as in blends, for their effects on parasitoid olfactory responses under laboratory conditions. Finally, two-choice cage experiments with plants treated with a behaviourally active synthetic blend versus control plants were performed to assess its attractive potential under greenhouse conditions. The cell-free cultivation medium of one of the attractive bacterial strains was included to evaluate the performance of the synthetic blend in comparison with the original bacterial culture.

\section{MATERIALS AND METHODS}

\subsection{Study organisms}

Three bacterial isolates that produce volatile blends that are attractive to A. colemani ${ }^{27,28}$ were used in this study (Table S1, Supporting Information). Strains were isolated from different sources from the habitat of Aphidius parasitoids. They included an isolate from 
the aphid Macrosiphum euphorbiae (ST18.16/150), an isolate from an Aphidius wasp (Aphidius ervi) (ST18.16/133), and an isolate from Dendrocerus aphidum, which is an hyperparasitoid of Aphidius (ST18.16/043). Bacteria were isolated from whole insects, which were homogenized with a motorized homogenizer (Precellys 24, Bertin Instruments) in $250 \mu \mathrm{l}$ sterile physiological water $(0.9 \% \mathrm{NaCl})$ with $0.01 \%$ Tween80 using $2 \mathrm{~mm}$ diameter glass beads. Homogenates were then plated on tryptic soy agar (TSA; Oxoid) supplemented with $0.3 \mathrm{~g} / \mathrm{L}$ cycloheximide to prevent fungal growth, and incubated at $25^{\circ} \mathrm{C}$. Based on sequencing of the $r p o B$ gene, isolates were assigned to Bacillus circulans (ST18.16/150), Bacillus pumilus (ST18.16/133) and Bacillus sp. (ST18.16/043) (Table S1, Supporting Information). Strains were stored at $-80^{\circ} \mathrm{C}$ in tryptic soy broth (TSB, Oxoid, Hampshire, UK) containing 25\% (v/v) glycerol. Insect responses were investigated using adult females of $A$. colemani. Parasitoids were obtained in the form of parasitized aphid mummies from Biobest (Westerlo, Belgium) (Aphidiussystem $\left.^{\circledR}\right)$. Mummies were placed inside a nylon insect cage $(20 \times 20 \times 20 \mathrm{~cm}$, BugDorm, MegaView Science Co., Ltd., Taichung, Taiwan) and kept under controlled conditions $\left(22^{\circ} \mathrm{C}, 70 \%\right.$ relative humidity and a 16:8-h light:dark photoperiod) until parasitoid emergence. All experiments were performed with food- and water-inexperienced females that were $<24$ hours old.

\section{$2.2 \quad$ Production of mVOCs}

For production of mVOCs, the procedure by Goelen et al. ${ }^{27}$ was used. Briefly, bacterial strains were plated on tryptic soy agar (TSA, Oxoid, Hampshire, UK) and incubated at 
$25^{\circ} \mathrm{C}$ for $24 \mathrm{~h}$. Next, bacterial cells were inoculated in $10 \mathrm{~mL}$ TSB and incubated overnight at $25^{\circ} \mathrm{C}$ with agitation at $120 \mathrm{rpm}$. Bacterial cells were then washed and diluted to a cell suspension with an optical density (OD $600 \mathrm{~nm}$ ) of 1 . Next, $1.5 \mathrm{~mL}$ of the obtained suspension was inoculated in a $250 \mathrm{~mL}$ Erlenmeyer flask containing $150 \mathrm{~mL}$ GYP25 medium ${ }^{27}$. Erlenmeyer flasks were sealed with silicone plugs and incubated at $25^{\circ} \mathrm{C}$ at $120 \mathrm{rpm}$. Each strain was cultivated in triplicate, and non-inoculated, blank medium was included as a control. After $48 \mathrm{~h}$ of incubation, the media were centrifuged at $10,000 \mathrm{~g}$ for 15 min and filter-sterilized to obtain cell-free supernatants. The samples were then stored in small aliquots in sterile, amber glass vials at $-20^{\circ} \mathrm{C}$ until further use.

\subsection{Identification of physiologically active mVOCs}

In order to determine which mVOCs elicited an electrophysiological response in $A$. colemani, first microbial volatiles were collected by dynamic headspace collection (air entrainment). ${ }^{29}$ Specifically, volatiles were collected for $1 \mathrm{~h}$ from $150 \mu \mathrm{L}$ cell-free cultivation medium inside a $4 \mathrm{~mL}$ glass screw top GC vial (Thermo Scientific, Waltham, USA). In- and outlet ports were created by fitting Swagelock ports onto 19Gx2” syringe needles (AganiTM, Terumo ${ }^{\circledR}$, Leuven, Belgium) which were pierced through the $12 \mathrm{~mm}$ polytetrafluorethylene (PTFE)/silicone septum (Supelco, Bellefonte, USA) of the GC vial. Activated charcoal filtered air was supplied through the inlet port at a rate of 400 $\mathrm{mL} / \mathrm{min}$. Air subsequently passed over the medium in the GC vial and headspace volatiles were adsorbed on Porapak Q filters (0.05 g, 50/80 mesh; Supelco, Bellefonte, USA) that were fitted on the outlet port through which air was drawn at a rate of $300 \mathrm{~mL} / \mathrm{min}$. Prior 
to entrainment, Porapak Q filters were washed with diethyl ether and conditioned by heating to $132^{\circ} \mathrm{C}$ in an activated charcoal-filtered nitrogen stream for $2 \mathrm{~h}$. Air entrainment of $150 \mu \mathrm{L}$ of blank GYP25 medium was included as a control. All connections in the air entrainment setup were made using PTFE tubing. Entrained volatiles were eluted in 750 $\mu \mathrm{L}$ diethyl ether and were stored in $1.1 \mathrm{~mL}$ glass microvials at $-20^{\circ} \mathrm{C}$ until further use. In line with previous work ${ }^{27,28}$, GC-FID analysis yielded highly similar mVOC profiles across the biological replicates for each treatment. Therefore, all remaining experiments (GC-EAG and behavioural assays, see below) were performed with only one of the three replicates.

After air entrainment, coupled gas chromatography-electroantennography (GCEAG) was performed using antennal preparations of female parasitoids. Before analysis, air entrainment samples were concentrated to $50 \mu \mathrm{L}$ under an activated charcoal-filtered nitrogen stream. GC-EAG analyses were performed three times, and for each replicate a new antennal preparation was used. The GC-EAG system was equipped with a $6890 \mathrm{~N}$ GC machine (Agilent Technologies, Santa Clara, USA) fitted with a cold on-column injection system and a non-polar HP-1 capillary column (50 m; $0.32 \mathrm{~mm}$ internal diameter; $0.52 \mu \mathrm{m}$ film thickness), and used a flame ionization detector (FID). ${ }^{30}$ The carrier gas was helium. The oven temperature was initiated at $30^{\circ} \mathrm{C}$ and was maintained there for $2 \mathrm{~min}$ before being raised to $250^{\circ} \mathrm{C}$ at a rate of $5^{\circ} \mathrm{C} / \mathrm{min}$. The $\mathrm{GC}$ column effluent was split equally between the FID and the heated transfer line which delivered the separated compounds into an activated charcoal filtered, humidified air stream that flew towards the antennal preparation. Antennal preparations were made by chilling the 
parasitoid in ice for 1 min, excising the head, removing one entire antenna, and then removing the tip of the last antennal segment to ensure good contact with the recording electrode. The antenna was then brought into contact with the Ag-AgCl ground electrode by inserting the antennal base into a glass capillary housing the electrode and filled with saline solution (composition as in Maddrell ${ }^{31}$, but without the glucose). The distal end was brought into contact with the recording electrode in a similar way. Detected signals were amplified by a high impedance amplifier (UN-06; Ockenfels Syntech GmbH, Kirchzarten, Germany) and analysed using customized Syntech software. Outputs from the FID and the EAG amplifier were analysed simultaneously with custom software. Only volatiles with a consistent electrophysiological response peak in all three replicates were considered as EAG-active.

Next, EAG-active mVOCs were tentatively identified by coupled GC-MS using 4 $\mu \mathrm{L}$ of the concentrated air entrainment samples on a Waters Autospec Ultima mass spectrometer (Manchester, UK) coupled to an Agilent 6890 GC (Agilent Technologies, Santa Clara, USA; cold on-column injector, $50 \mathrm{~m} \times 0.32 \mathrm{~mm}$ internal diam, $0.52 \mu \mathrm{m}$ film thickness HP-1- column). Ionization was performed by electron impact at $70 \mathrm{eV}$ and $220^{\circ} \mathrm{C}$. The GC oven temperature was initiated at $30^{\circ} \mathrm{C}$ and maintained for $5 \mathrm{~min}$ and then raised to $250^{\circ} \mathrm{C}$ at $5^{\circ} \mathrm{C} / \mathrm{min}$. Helium was the carrier gas. Peak identities were tentatively determined by manually comparing mass spectra with those from mass spectral databases using NIST MS Search v2.0 software with the NIST 2011 library, and by comparison of GC retention indices (Kováts index = KI). 


\section{$2.4 \quad$ Y-tube behavioural assays}

In order to test the attractiveness of the microbial volatile blends and EAG-active volatiles or blends thereof, a Y-tube olfactometer bioassay was performed as described by Goelen et al. ${ }^{27}$ Specifically, a glass Y-tube olfactometer (base: $20 \mathrm{~cm}$; arms:12 cm with a $60^{\circ}$ angle at the $\mathrm{Y}$-junction; inner diameter: $1.5 \mathrm{~cm}$ ), connected to an air pump producing an unidirectional airflow of $400 \mathrm{ml} \mathrm{min}^{-1}$ from the arms to the base, was put on a table that was homogeneously illuminated by four $24 \mathrm{~W}$ T5 TL-fluorescent tubes $(16 \times 549 \mathrm{~mm}$, 1350 Lumen, $5500 \mathrm{~K}$ ) at a height of $45 \mathrm{~cm}$. To avoid any visual distraction of the parasitoid wasps, a white curtain was placed around the olfactometer. To improve parasitoid responsiveness, the olfactometer was mounted at a $20^{\circ}$ incline, by which the insects were stimulated to move towards the bifurcation. For each bacterial strain, $150 \mu \mathrm{L}$ of the cell-free cultivation medium was loaded on a filter paper (37 mm; Macherey-Nagel, Düren, Germany) and subsequently put in one of the olfactometer odour chambers. The second chamber received another filter on which $150 \mu \mathrm{L}$ blank medium was loaded as a control. For assessing parasitoid response to EAG-active compounds, benzaldehyde ( $\geq 99.5 \%$ ), butyl acetate (99.7\%), 1,3-diacetyl benzene (97.0\%), styrene ( $\geq 99.0 \%$ ) (all purchased from Sigma-Aldrich, Saint Louis, USA) and 1,2-dimethyl benzene (o-xylene) ( $\geq 99.0 \%$ Fluka, Bucharest, Romania) were used. Compounds were dissolved in diethyl ether prior to loading $10 \mu \mathrm{L}$ of the mixture on a filter paper. After 30 seconds (which allowed the diethyl ether to evaporate), the filter paper was placed in one of the odour chambers of the olfactometer setup, while in the other chamber another filter paper was placed on which $10 \mu \mathrm{L}$ diethyl ether had been added as a control. In a first experiment, 
the different test compounds were diluted in diethyl ether in different concentrations, resulting in seven different doses, i.e. 1, 10, 50 and 100 ng, and 1, 10 and $50 \mu$ g, which were then each tested in the Y-tube olfactometer. In a second experiment, two synthetic volatile blends were tested, which are further referred to as "Blend 1" and "Blend 2". Blend 1 consisted of two compounds to which A. colemani showed significant preference in the first experiment, i.e. benzaldehyde and styrene. The blend was produced by combining both compounds in their most attractive dose as determined in the first experiment (i.e. $10 \mathrm{ng}$ for benzaldehyde and $1 \mu \mathrm{g}$ for styrene). In addition, four other doses of the blend were tested with the same ratio of both compounds (Table S2, Supporting Information). Blend 2 consisted of five EAG-active compounds and was created by adding the different compounds at relative amounts resembling the ratios in the mVOC blend of one of the bacterial strains (ST18.16/133), and was tested at five different doses (Table S3, Supporting Information).

All experiments were conducted with 60 female individuals, which were released in twelve cohorts of five individuals, and olfactory response was evaluated 10 min after their release. Parasitoids that did not make a choice within 10 min after release were considered as non-responding individuals and were eliminated from statistical analysis. For every release, new parasitoid females were used. The filter papers inside the odour chambers were renewed after every two releases. Additionally, the arms of the Y-tube olfactometer were flipped $180^{\circ}$ after each six releases to minimize any spatial effects on parasitoid choice. At the same time, the Y-tube was also renewed by a cleaned Y-tube. At the end of the assay, all olfactometer parts were rinsed with tap water, distilled water, 
acetone and finally pentane, after which the parts were kept overnight at $150^{\circ} \mathrm{C}$. All bioassays were conducted at $21 \pm 2^{\circ} \mathrm{C}, 60 \pm 5 \% \mathrm{RH}$ and performed between 09:00 and 16:00. Parasitoid olfactory response was analysed using a Generalized Linear Mixed Model (GLMM) based on a binomial distribution with a logit link function (logistic regression) using bacterial isolate, compound or blend as fixed factor (performed in $\mathrm{R}$ with the 'glmer' function from the lme4 package). Each release of one cohort of five individuals served as a replicate, giving a total of 12 replicates. To adjust for overdispersion and to prevent pseudoreplication, the release of each cohort of five individuals $(n=12)$ was included in the model as a random factor. The number of parasitoids choosing the control or treatment side in each cohort was entered as response variable. To examine the preference of the investigated parasitoids, we tested the null hypothesis (H0) that the parasitoids showed no preference for any olfactometer arm (i.e. 50:50 response) by testing $\mathrm{H}_{0}$ : logit $=0$, which equals a 50:50 distribution. Results were presented by calculating the Preference Index (PI), which is the difference between the number of parasitoids choosing the volatile compounds and the parasitoids choosing the control divided by the total number of responding insects.

\subsection{Cage experiments}

Following the laboratory bioassays, the most attractive blend (i.e. Blend 1 at a dose of 1 $\mathrm{ng} / \mu \mathrm{L}$ benzaldehyde and $100 \mathrm{ng} / \mu \mathrm{L}$ styrene) was tested in a two-choice cage experiment in a greenhouse compartment (average temperature $22 \pm 4^{\circ} \mathrm{C}$, day light). As a comparison, the cell-free cultivation medium of one of the attractive bacterial strains (ST18.16/133) 
was included. Experiments were performed in a $2 \times 3 \times 2 \mathrm{~m}$ cage that was closed at all sides with a fine mesh. Nine-week-old sweet pepper plants (Capsicum annuum cv. IDS) were placed onto elevated platforms (height: $40 \mathrm{~cm}$ ) in each corner of the cage (one plant in each corner) (Fig. S1, Supporting Information). Using a vaporizer, plants were treated by spraying them with either the synthetic blend of $1 \mathrm{ng} / \mu \mathrm{L}$ benzaldehyde and $100 \mathrm{ng} / \mu \mathrm{L}$ styrene (Blend 1) or the cell-free cultivation medium of strain ST18.16/133 (“Treatment”; two plants), or a control solution (diethyl ether when the synthetic blend was tested or non-inoculated GYP25 medium when the bacterial cultivation medium was tested) (“Control”; two plants). Specifically, the leaves of the plants were sprayed with 20 puffs by which on average $2.5 \mathrm{~mL}$ was deposited onto the leaves of each plant. Treatment and control plants were always placed diagonally relative to each other. To evaluate the ability of the volatile mixtures to affect the behavioural response of $A$. colemani, 60 females were released from an elevated platform (height: $40 \mathrm{~cm}$ ) in the centre of the cage $30 \mathrm{~min}$ after the plants had been sprayed (Fig. S1, Supporting Information). To record the parasitoids' responses, a transparent, non-odorous glue plate $(40 \times 25 \mathrm{~cm}$; Biobest, Westerlo, Belgium) was placed directly behind each plant to trap the parasitoids that visited this part of the cage (Fig. S1, Supporting Information). Forty-eight hours after parasitoid release, traps were removed and trapped parasitoids were counted. The experiment was replicated eight times on four different experimental days. For each replicate, plants were renewed, and the positions of treatment and control plants were switched. Parasitoid behavioural response was analysed as mentioned earlier using a GLMM based on a binomial distribution with a logit link function (logistic regression), 
but using blend (synthetic blend vs. bacterial culture medium) as fixed factor. Each release of 60 individuals served as a replicate. The total number of parasitoids choosing the control or treatment plants in each replicate was entered as response variable.

\section{$3 \quad$ RESULTS}

\subsection{Electrophysiological responses of $A$. colemani to mVOCs}

In total, 20 EAG-active compounds were found in the mVOCs released by the bacteria (Fig. S2, Supporting Information), nine of which were tentatively identified by GC-MS and KI comparison (Table 1). While most of the EAG-responses were elicited by compounds unique to a certain strain, five EAG-active compounds originated from the mVOCs of more than one strain (Table 1). Specifically, the EAG-active compounds styrene and $o$-xylene were found in the volatile extracts of the three investigated strains, while benzaldehyde, 1,3-diacetylbenzene and an unidentified compound were found in the volatile blends produced by two strains (Table 1).

\subsection{Olfactory responses to EAG-active compounds and blends thereof}

Behavioural assays with five selected EAG-active compounds revealed that parasitoids showed a significant behavioural response to two compounds: styrene and benzaldehyde (Fig. 1). Compound dose significantly affected parasitoid response (styrene: $\chi^{2}=23.33$, $d f=6, P=0.003$; benzaldehyde: $\left.\chi^{2}=18.73, d f=6, P=0.016\right)$. Parasitoids had a significant preference for styrene at $1 \mu \mathrm{g}$ dose $(\mathrm{PI}=0.38, P=0.005)$, and for 
benzaldehyde at $50 \mathrm{ng}(\mathrm{PI}=0.29, P=0.035)$ and $10 \mathrm{ng}(\mathrm{PI}=0.31, P=0.011)$ doses (Fig. 1). Olfactory response to 10 or 50 ng benzaldehyde was comparable with the response to the bacterial cultivation medium (PI $=0.30-0.33$ ), while the response to $1 \mu \mathrm{g}$ styrene was more pronounced (Fig. 1). Results for benzaldehyde also suggest that doses equal or higher than $1 \mu \mathrm{g}$ elicit a negative response in A. colemani. Furthermore, results revealed that $10 \mathrm{ng}$ of butyl acetate was significantly repellent to $A$. colemani $(\mathrm{PI}=-0.36 ; P=$ 0.011) (Fig. 1).

Of the two synthetic blends tested, parasitoids were significantly attracted to Blend $1\left(\chi^{2}=21.15, d f=4, P<0.001\right)$, while the effect of Blend 2 was not significant in any of the doses tested $\left(\chi^{2}=5.90, d f=4, P=0.207\right)$ (Fig. 2). Parasitoid females had a significant preference for the $0.75 \times(\mathrm{PI}=0.32 ; P=0.043), 1 \times(\mathrm{PI}=0.50, P<0.001)$ and $1.5 \times$ dose (PI $=0.28, P=0.022)$ of Blend 1 , while they were significantly deterred by the

$2 \times$ dose $(\mathrm{PI}=-0.28, P=0.046)($ Fig. 2$)$. A combination of $1 \mu \mathrm{g}$ styrene and $10 \mathrm{ng}$ benzaldehyde elicited a considerably stronger response $(\mathrm{PI}=0.50)$ in comparison to the responses to the individual compounds $\left(\mathrm{PI}_{\text {styrene }}=0.38, \mathrm{PI}_{\text {benzaldehyde }}=0.31\right)$ and the mVOCs of the bacterial cell-free media (PI $=0.30-0.33)$.

\subsection{Parasitoid behavioural response under greenhouse conditions}

Parasitoid behavioural response in the two-choice cage experiment varied significantly between synthetic Blend 1 and the cell-free cultivation medium of strain ST18.16/133 $\left(\chi^{2}\right.$ $=5.75, \mathrm{df}=4, P=0.016)$. Plants treated with Blend 1 were visited by significantly more parasitoids than the control plants ( $\mathrm{PI}=0.35, P<0.001$ ), while plants treated with the 
cultivation medium of ST18.16/133 elicited no significant response relative to the control plants (PI $=0.03, P=0.677$ ) (Fig. 3). In the experiment with Blend 1, on average $13.5 \pm$ 1.6 (SEM) parasitoids were caught on plates near the treated plants, while $6.5 \pm 1.1$ parasitoids were caught on the plates near the control plants. In the second experiment where plants were treated with the cultivation medium of ST18.16/133 on average $6.0 \pm$ 0.8 parasitoids were caught near the treated plants, while $5.5 \pm 0.7$ parasitoids were trapped on the plates near the plants that received the control medium. It has to be noted that our method used to evaluate insect response may have underestimated the number of responding parasitoids as only individuals trapped on the glue plates behind the plants were taken into account.

\section{DISCUSSION}

Our results showed that $A$. colemani females were able to detect several, but not all, mVOCs produced by the bacteria. This suggests that only certain mVOCs play a role in parasitoid olfactory behaviour, which is in agreement with previous research on plant- or host-associated volatiles. ${ }^{21}$ Although GC-EAG analyses allow the determination of electrophysiologically active compounds, an EAG response does not necessarily indicate behavioural activity. ${ }^{32}$ In our study, only two of five tested EAG-active compounds, i.e. benzaldehyde and styrene, evoked an innate behavioural response in the Y-tube bioassays. Styrene was produced by the three bacterial strains tested, while benzaldehyde was produced by ST18.16/133 and ST18.16/150, but not by ST18.16/043. Further, the olfactory response varied in a dose-dependent manner, ranging from no or negative 
responses to positive responses. This has previously been observed for HIPVs in braconid parasitoids. ${ }^{25,33}$ Interestingly, styrene at a dose of $1 \mu \mathrm{g}$ and benzaldehyde at $10 \mathrm{ng}$ or 50 ng doses elicited a similar or even stronger positive response in A. colemani than the cellfree cultivation medium of the bacteria. Similar findings have been reported for Psyttalia parasitoids, which were more or equally attracted to individual synthetic Ceratitis capitata-induced fruit volatiles than to the odour of infested fruits themselves. ${ }^{34}$ The higher sensitivity to benzaldehyde compared to styrene suggests it is a more ecologically relevant compound. Benzaldehyde is widely emitted by plants, and flowers in particular, ${ }^{35}$ which may explain the high preference observed for this compound. Bacterial VOC blends are generally composed of typical fermentation products such as methylated, low molecular weight alcohols and corresponding aldehydes and organic acids. ${ }^{20,36}$ However, some compounds emitted by microbes are also commonly reported as plant volatiles or insect pheromones. ${ }^{27}$ It is therefore possible that the parasitoids were attracted to benzaldehyde in the context of it being a floral volatile rather than coincidental production by bacteria as side-products of their primary and secondary metabolism. ${ }^{37}$ However, recent findings have shown that many mVOCs are not simply side-products, but display certain biological activities, ${ }^{38}$ e.g. to aid microbial dispersal by insect vectors. ${ }^{39,40}$ Strikingly, parasitoids were equally attracted to the mVOC blends of the three bacterial strains investigated. For bacteria isolated from hyperparasitoids, this result is counterintuitive as optimal foraging theory assumes that insects are only attracted to signals from which they benefit the most for the lowest cost. So far, it is still unknown why A. colemani responds to mVOCs from bacteria isolated from hyperparasitoids, but a recent study 
suggests that bacterial phylogeny rather than source of isolation is the main driver behind parasitoid olfactory behaviour. ${ }^{27}$ Because microorganisms represent a minor fraction of biomass, it remains also unclear to what extent mVOCs contribute to parasitoid foraging behaviour in agricultural fields, which typically represent complex odour environments. Further research is needed to unravel the precise ecological role of volatiles produced by bacteria, and to what extent they affect plant-insect interactions.

Although no behavioural responses were observed for a number of EAG-active compounds, or specific concentrations of EAG-active compounds, it has to be noted that these compounds or concentrations may still exert an effect within a blend of volatiles. Previous research has demonstrated that insects that are attracted to a specific blend can be unaffected by or even repelled by the individual compounds of that blend. ${ }^{22,41}$ In addition, it has to be considered that the parasitoids used in this study had not been previously exposed to the mVOCs tested. It is possible that compounds that did not elicit an innate response in our studies, may elicit a conditioned response as a result of associative learning, when parasitoids experience these volatiles in association with feeding or oviposition events. ${ }^{42}$

Parasitoids were not only attracted by individual compounds, but also by mixtures of synthetic mVOCs. Specifically, a strong positive response was observed for a synthetic mixture of styrene and benzaldehyde when combined at a ratio of 100/1. Moreover, at a dose of $1 \mu \mathrm{g}$ styrene and $1 \mathrm{ng}$ benzaldehyde, parasitoid preference for the blend was considerably higher than for the individual compounds. At these amounts, the blend attracted $75 \%$ of the responding individuals $(\mathrm{PI}=0.50)$, which is comparable to levels of 
positive response obtained with synthetic plant volatiles and volatiles from aphid-infested plants in Aphidius species. ${ }^{33,43}$ Additionally, A. colemani response to our two-component blend was also stronger than to a bacterial cultivation medium, which suggests that the latter may contain compounds that have a masking or inhibitory effect on the key compounds responsible for the attractiveness of the blend. ${ }^{44 ; 45}$ These findings could also be interpreted as an indication that parasitoids had an innate response to simple blends with typical floral volatiles like benzaldehyde. ${ }^{35}$ Several examples exist where the response to a blend containing a select number of synthetic compounds exceeded the response to the natural blend. ${ }^{44,46}$

By contrast, the synthetic mixture of EAG-active compounds mimicking the behaviourally active cultivation medium of bacterial strain ST18.16/133 did not induce a positive behavioural response in $A$. colemani, despite the presence of styrene and benzaldehyde in the mixture. However, the amounts and proportions of styrene and benzaldehyde in this blend were different compared to the active two-compound blend. It is also possible that one or more key compounds that were present in the bacterial cultivation medium were absent in the synthetic mixture of five compounds. Previous research has shown that removing key compounds from an attractive volatile blend can disrupt attraction to that blend. ${ }^{47}$ It is therefore possible that one or more of the unidentified EAG-active compounds in the bacterial volatile emissions have been essential in eliciting the attractive response in A. colemani. Additional research is required to identify these EAG-active compounds and assess their effects on parasitoid olfactory response, both individually and in mixtures. 
In contrast to the laboratory assays, the cell-free cultivation medium of strain ST18.16/133 did not show significant attraction of A. colemani in the cage experiments. This confirms previous research showing that results from laboratory experiments cannot always be extrapolated to more realistic environments and over longer distances. ${ }^{46,48}$ Under natural conditions, there are more complex background odours originating from diverse sources which can compete or interact with attractants, thereby reducing the signal-to-noise ratio and interfering with the insect's response. ${ }^{46,49}$ In contrast, application of the two-component mixture of styrene and benzaldehyde resulted in significant attraction of the parasitoids to the treated plants. Parasitoid responsiveness to the synthetic blend was also significantly higher compared to the cell-free cultivation medium, further demonstrating the attractiveness of the two-compound blend. However, to unambiguously show the attractiveness of the synthetic blend, experiments should also be conducted with infested plants. Furthermore, research is needed to establish whether the observed effects were directly caused by the applied blend of synthetic volatiles, or whether they were the result of an interaction between the applied compounds and the plants, inducing the production of attractive volatiles.

\section{CONCLUSIONS}

In conclusion, this study demonstrated that mVOCs emitted by bacteria elicited behavioural and electrophysiological responses in A. colemani parasitoids. The olfactory response of $A$. colemani to synthetic blends based on bacterial volatile emissions was largely dependent on the dose and ratio of the different compounds. Moreover, synthetic 
volatile blends were able to attract $A$. colemani parasitoids under greenhouse conditions, while this was not the case for the more complex bacterial cell-free cultivation medium. This opens opportunities to construct simple synthetic blends to attract or retain natural enemies of pest species at the greenhouse or field scale. Future research is needed to assess whether attracting natural enemies with such compounds will also enhance biological control efficacy.

\section{ACKNOWLEDGEMENTS}

We would like to thank the Flemish Fund for Scientific Research (FWO; 1S15116316N) and Flanders Innovation \& Entrepreneurship (VLAIO; HBC.2018/2022) for financial support. Further, we are grateful to Dries Belmans and Francine van Neerbos for help with the experiments, and Sofie Bossaert for help with the figures.

\section{CONFLICT OF INTEREST DECLARATION}

All authors declare that no competing interests exist.

\section{REFERENCES}

1 Bale JS, van Lenteren JC and Bigler F, Biological control and sustainable food production. Philos Trans R Soc Lond B Biol Sci 363:761-776 (2008). 
2 Tscharntke T, Karp DS, Chaplin-Kramer R, Batárya P, DeClerck F, Grattone C et al., When natural habitat fails to enhance biological pest control - Five hypotheses. Biol Conserv 204:449-458 (2016).

3 van Lenteren JC, The state of commercial augmentative biological control: plenty of natural enemies, but a frustrating lack of uptake. BioControl 57:1-20 (2012).

4 Kaplan I, Attracting carnivorous arthropods with plant volatiles: The future of biocontrol or playing with fire? Biol Control 60:77-89 (2012).

5 Turlings TCJ and Erb M, Tritrophic interactions mediated by herbivore-induced plant volatiles: mechanisms, ecological relevance, and application potential. Annu Rev Entomol 63:433-452 (2018).

6 Witzgall $\mathrm{P}$, Kirsch $\mathrm{P}$ and Cork A, Sex pheromones and their impact on pest management. J Chem Ecol 36:80-100 (2010).

$7 \quad$ Picket JA and Khan ZR, Plant volatile-mediated signalling and its application in agriculture: successes and challenges. New Phytol 212:856-870 (2016).

8 Leroy PD, Sabri A, Verheggen FJ, Francis F, Thonart P and Haubruge E, The semiochemically mediated interactions between bacteria and insects. Chemoecol 21:113-122 (2011).

9 Davis TS, Crippen TL, Hofstetter RW and Tomberlin JK, Microbial volatile emissions as insect semiochemicals. J Chem Ecol 39:840-859 (2013).

10 Ali JG, Casteel CL, Mauck KE and Trase O, Chemical ecology of multitrophic microbial interactions: plants, insects, microbes and the metabolites that connect them. J Chem Ecol 46:645-648 (2020). 
11 Francis F, Jacquemyn H, Delvigne F and Lievens B, From diverse origins to specific targets: role of microorganisms in indirect pest biological control. Insects 11:533 (2020).

12 Becher PG, Flick G, Rozpędowska E, Schmidt A, Hagman A, Lebreton S et al., Yeast, not fruit volatiles mediate Drosophila melanogaster attraction, oviposition and development. Fun Ecol 26:822-828 (2012).

13 Leroy PD, Sabri A, Heuskin S, Thonart P, Lognay G, Verheggen FJ et al., Microorganisms from aphid honeydew attract and enhance the efficacy of natural enemies. Nat Comm 2:348 (2011)

14 Sobhy IS, Baets D, Goelen T, Herrera-Malaver B, Bosmans L, Van den Ende W et al., Sweet scents: nectar specialist yeasts enhance nectar attraction of a generalist aphid parasitoid without affecting survival. Front Plant Sci 9:1009 (2018).

15 Stensmyr MC, Dweck HKM, Farhan A, Ibba I, Strutz A, Mukunda L et al., A conserved dedicated olfactory circuit for detecting harmful microbes in Drosophila. Cell 1511:1345-1357 (2012).

16 Scheidler NH, Liu C, Hamby KA, Zalom FG and Syed Z, Volatile codes: correlation of olfactory signals and reception in Drosophila-yeast chemical communication. Sci Rep 5:14059 (2015).

17 Rering CC, Beck JJ, Hall GW, McCartney MM and Vannette RL, Nectarinhabiting microorganisms influence nectar volatile composition and attractiveness to a generalist pollinator. New Phyt 220:655-658 (2018). 
18 Sobhy IS, Goelen T, Herrera-Malaver B, Verstrepen KJ, Wäckers F, Jacquemyn et al., Associative learning and memory retention of nectar yeast volatiles in a generalist parasitoid. Anim Behav 153: 137-146 (2019).

19 Fischer CY, Detrain C, Thonart P, Haubruge E, Francis F, Verheggen FJ et al., Bacteria may contribute to distant species recognition in ant-aphid mutualistic relationships. Insect Sci 24:278-284 (2017).

20 Dzialo MC, Park R, Steensels J, Lievens B and Verstrepen KJ, Physiology, ecology and industrial applications of aroma formation in yeast. FEMS Microbiol Rev 41:S95-S128 (2017).

21 Bruce TJA, Wadhams LJ and Woodcock CM, Insect host location: a volatile situation. Trends in Plant Sci 10:269-274 (2005).

22 Van Wijk M, De Bruijn PJ and Sabelis MW, Complex odor from plants under attack: Herbivore's enemies react to the whole, not its parts. PLoS ONE 6:e21742 (2011).

23 Wei JN, Wang L, Zhu J, Zhang S, Nandi OI and Kang L, Plants attract parasitic wasps to defend themselves against insect pests by releasing hexenol. PLOS ONE 2:e852 (2007).

24 Ye M, Veyrat N, Xu H, Hu L, Turlings TCJ and Erb M, An herbivore-induced plant volatile reduces parasitoid attraction by changing the smell of caterpillars. Sci Adv 4:eaar4767 (2018). 
25 Shiojiri K, Ozawa R, Kugimiya S, Uefune M, van Wijk M, Sabelis MW et al., Herbivore-specific, density-dependent induction of plant volatiles: honest or “cry wolf” signals? PLoS ONE 5:e12161 (2010).

26 Uefune M, Kugimiya S, Ozawa R and Takabayashi J, Parasitic wasp females are attracted to blends of host-induced plant volatiles: do qualitative and quantitative differences in the blend matter? F1000Research 2:57 (2013).

27 Goelen T, Sobhy IS, Vanderaa C, de Boer JG, Delvigne F, Francis F et al., Volatiles of bacteria associated with parasitoid habitats elicit distinct olfactory responses in an aphid parasitoid and its hyperparasitoid. Fun Ecol 34:507-520 (2020).

28 Goelen T, Sobhy IS, Vanderaa C, Wäckers F, Rediers H, Wenseleers T et al., Bacterial phylogeny predicts volatile organic compound composition and olfactory response of an aphid parasitoid. Oikos 129:1414-1428 (2020).

29 Birkett MA, The chemistry of plant signalling, in Plant communication from an ecological perspective, ed. by Baluška F and Ninkovic V, Springer, BerlinHeidelberg, pp. 21-42 (2010).

30 Wadhams LJ, The use of coupled gas chromatography: electrophysiological techniques in the identification of insect pheromones, in Chromatography and isolation of insect hormones and pheromones, ed. by McCaffery AR and Wilson ID, Plenum, New York, pp. 289- 298 (1990).

31 Maddrell SHP, Secretion by the Malphigian tubules of Rhodnius. The movement of ions and water. J Exp Biol 51:71-97 (1969). 
32 Park KC, Zhu J, Harris J, Ochieng SA and Baker TC, Electroantennogram responses of a parasitic wasp, Microplitis croceipes, to host-related volatile and anthropogenic compounds. Physiol Entomol 26:69-77 (2001).

33 Takemoto $\mathrm{H}$ and Takabayashi J, Parasitic wasps Aphidius ervi are more attracted to a blend of host-induced plant volatiles than to the independent compounds. $J$ Chem Ecol 41:801-807 (2015).

34 Benelli G, Revadi S, Carpita A, Giunti G, Raspi A, Anfora G et al., Behavioral and electrophysiological responses of the parasitic wasp Psyttalia concolor (Szépligeti) (Hymenoptera: Braconidae) to Ceratitis capitata-induced fruit volatiles. Biol Control 64:116-124 (2013).

35 Knudsen JT, Tollsten L and Bergström LG, Floral scents - a checklist of volatile compounds isolated by head-space techniques. Phytochem 33:253-280 (1993).

36 Schmidt R, Cordovez V, de Boer W, Raaijmakers J and Garbeva P, Volatile affairs in microbial interactions. Int Soc Microbial Ecol J 9:2329-2335 (2015).

37 Korpi A, Järnberg J and Pasanen A, Microbial volatile organic compounds. Crit Rev Toxicol 39:139-193 (2009).

38 Sugio A, Dubreuil G, Giron D and Simon J-C, Plant-insect interactions under bacterial influence: ecological implications and underlying mechanisms, $J$ Exp Bot 66:467-478 (2015).

39 Christiaens JF, Franco LM, Cools TL, De Meester L, Michiels J, Wenseleers T et al., The fungal aroma gene ATF1 promotes dispersal of yeast cells through insect vectors. Cell Reports 9: 425-432 (2014). 
40 Mann RS, Ali JG, Hermann SL, Tiwari S, Pelz-Stelinski KS, Alborn HT et al., Induced release of a plant-defense volatile ‘deceptively’ attracts insect vectors to plants infected with a bacterial pathogen. PLoS Pathog 8:e1002610 (2012).

41 Webster B, Bruce T, Pickett J and Hardie J, Volatiles functioning as host cues in a blend become nonhost cues when presented alone to the black bean aphid. Anim Behav 79:451-457 (2010).

42 Meiners T, Wäckers F and Lewis WJ, Associative learning of complex odours in parasitoid host location. Chem Senses 28:231-236 (2003).

43 Yang S, Xu R, Yang SY and Kuang RP, Olfactory responses of Aphidius gifuensis to odors of host plants and aphid-plant complexes. Insect Sci 16:503-551 (2009).

44 Cha DH, Adams T, Werle CT, Sampson BJ, Adamczyk Jr JJ, Rogg H et al., A four-component synthetic attractant for Drosophila suzukii (Diptera: Drosophilidae) isolated from fermented bait headspace. Pest Manag Sci 70:324331 (2014).

45 Verschut TA, Carlsson MA and Hambäck PA, Scaling the interactive effects of attractive and repellent odours for insect search behaviour. Sci Rep 9:1-8 (2019).

46 Cai X, Bian L, Xu X, Luo Z, Li Z and Chen Z, Field background odour should be taken into account when formulating a pest attractant based on plant volatiles. Sci Rep 7:41818 (2017).

47 Tasin M, Bäckman AC, Coracini M, Casado D, Ioriatti C and Witzgall P, Synergism and redundancy in a plant volatile blend attracting grapevine moth females. Phytochem 68:203-209 (2007). 
48 Knudsen GK, Bengtsson M, Kobro S, Jaastad G, Hofsvang T and Witzgall P, Discrepancy in laboratory and field attraction of apple fruit moth Argyresthia conjugella to host plant volatiles. Physiol Entomol 33:1-6 (2008).

49 Schröder R and Hilker M, The relevance of background odor in resource location by insects: a behavioral approach. BioScience 54:308-316 (2008). 
Table 1. Compounds ${ }^{a}$ identified by coupled GC-EAG, using female Aphidius colemani antennae, in volatile extracts collected from the cell-free cultivation medium of three bacterial strains that are attractive to A. colemani and the blank medium. Compounds in bold were selected for behavioural experiments.

\begin{tabular}{|c|c|c|c|c|c|c|c|}
\hline \multirow[b]{2}{*}{ EAG response ${ }^{\mathrm{b}}$} & \multirow[b]{2}{*}{$\mathrm{RT}(\min )$} & \multirow[b]{2}{*}{$\mathbf{R I}^{\mathbf{c}}$} & \multirow[b]{2}{*}{ Compound } & \multirow[t]{2}{*}{ Blank medium } & \multicolumn{3}{|c|}{ Attractive strains } \\
\hline & & & & & ST18.16/133 & ST18.16/043 & ST18.16/150 \\
\hline A1, D1 & 4.28 & 705 & heptane & 33.1 & & & 53.6 \\
\hline $\mathrm{C} 1$ & 4.57 & 727 & unknown 1 & & & 15.4 & \\
\hline B1 & 4.72 & 738 & unknown 2 & & 1.7 & & \\
\hline A2 & 4.77 & 741 & 2,4-dimethyl hexane & 1.4 & & & \\
\hline A3 & 5.35 & 780 & unknown 3 & 0.8 & & & \\
\hline B2 & 5.66 & 798 & butyl acetate & & 4.2 & & \\
\hline D2 & 6.17 & 837 & ethyl cyclohexane & & & & 1.1 \\
\hline D3 & 6.58 & 868 & cyclohexanone & & & & 18.9 \\
\hline B3, C2, D4 & 6.92 & 890 & styrene & & 1.2 & 0.8 & 1.3 \\
\hline B4, C3, D5 & 7.02 & 896 & o-xylene & & 2.2 & 3.2 & 6.0 \\
\hline C4 & 7.09 & 901 & unknown 4 & & & 0.9 & \\
\hline B5 & 7.42 & 929 & unknown 5 & & 1.0 & & \\
\hline B6, D6 & 7.50 & 935 & benzaldehyde & & 1.3 & & 1.6 \\
\hline B7, D7 & 7.75 & 956 & unknown 6 & & 4.6 & & 11.3 \\
\hline D8 & 8.02 & 976 & unknown 7 & & & & 0.7 \\
\hline A4 & 10.30 & 1175 & unknown 8 & 2.7 & & & \\
\hline B8 & 11.21 & 1263 & unknown 9 & & 1.1 & & \\
\hline $\mathrm{C} 5$ & 11.91 & 1335 & unknown 10 & & & 0.9 & \\
\hline B9, C6 & 12.53 & 1399 & 1,3-diacetylbenzene & & 1.2 & 3.4 & \\
\hline
\end{tabular}


'better and number combinations refer to the different panels and marked EAG-active peaks in Fig. S2 (Supporting Information).

${ }^{\mathrm{C}}$ Retention indices (Kováts index) relative to retention times of C7-C22 n-alkanes on an HP-1 GC column. 


\section{FIGURE LEGENDS}

Figure 1. Olfactory responses of adult Aphidius colemani females (tested in 12 cohorts of 5 females) when given the choice between one of seven different doses ranging from $1 \mathrm{ng}$ to $50 \mu \mathrm{g}$ of five synthetic volatile compounds (i.e. butyl acetate, styrene, $o$-xylene, benzaldehyde and 1,3-diacetylbenzene) and a diethyl ether blank in a Y-tube olfactometer bioassay. Olfactory response of $A$. colemani to the mVOCs of the bacterial strains ST18.16/133, ST18.16/043 and ST18.16/150 were included as a reference. Grey bars indicate non-significant olfactory responses $(P>0.05)$, blue bars indicate significant attractive responses $(P \leq 0.05)$ and red bars indicate significant repellent responses $(P \leq$ 0.05) when compared to a theoretical 50:50 distribution within a choice test (Generalized Linear Mixed Model). $* * 0.001 \leq P<0.01 ; * 0.01 \leq P \leq 0.05$; ns, non-significant. Overall parasitoid responsiveness was higher than $67 \%$.

Figure 2. Olfactory responses of adult Aphidius colemani females (tested in 12 cohorts of 5 females) when given the choice between one of five different doses of a synthetic volatile blend and a diethyl ether blank in a Y-tube olfactometer bioassay. Synthetic blends tested included (A) Blend 1, consisting of two compounds (benzaldehyde and styrene) and (B) Blend 2, consisting of five compounds (butyl acetate, o-xylene, benzaldehyde, styrene, and 1,3-diacetylbenzene). For Blend 1, dose 1× was composed of $1 \mu \mathrm{g}$ styrene and $10 \mathrm{ng}$ benzaldehyde; for Blend 2, dose $1 \times$ consisted of $3.40 \mu \mathrm{g}$ butyl acetate, $1.81 \mu \mathrm{g}$ o-xylene, $1.07 \mu \mathrm{g}$ benzaldehyde, $1.00 \mu \mathrm{g}$ styrene, and $0.98 \mu \mathrm{g}$ 1,3- 
diacetylbenzene. Olfactory response of $A$. colemani to the mVOCs of the bacterial strains ST18.16/133, ST18.16/043 and ST18.16/150 is included as a reference. Grey bars indicate non-significant olfactory responses $(P>0.05)$, blue bars indicate significant attractive responses $(P \leq 0.05)$ and red bars indicate significant repellent responses $(P \leq$ 0.05) when compared to a theoretical 50:50 distribution within a choice test (Generalized Linear Mixed Model). ${ }^{* * *} P<0.001 ; * 0.01 \leq P \leq 0.05$; ns, non-significant. Overall parasitoid responsiveness was higher than $80 \%$.

Figure 3. Responses of adult Aphidius colemani females under greenhouse conditions when given the choice between two sweet pepper plants treated with a volatile blend and two control plants ( $n=8$; per replicate 60 individuals were released). Experiments included application of (A) Blend 1 and diethyl ether as a control, and application of (B) the cell-free cultivation medium of ST18.16/133 and blank GYP25 medium as a control. Blend 1 was composed of $100 \mathrm{ng} / \mu \mathrm{L}$ styrene and $1 \mathrm{ng} / \mu \mathrm{L}$ benzaldehyde. Parasitoid response was evaluated $48 \mathrm{~h}$ after insect release by counting the number of trapped wasps on transparent, odourless glue plates behind the plants. The blue bar indicates an average significant attractive response ( $P \leq 0.05)$, while the grey bar indicates an average nonsignificant olfactory response $(P>0.05)$ when compared to a theoretical 50:50 distribution within a choice test (Generalized Linear Mixed Model). ${ }^{* * *} P<0.001$; ns, non-significant. Average responsiveness for Blend 1 was 33.0\%, for the ST18.16/133 culture medium it was $19.2 \%$. 
A
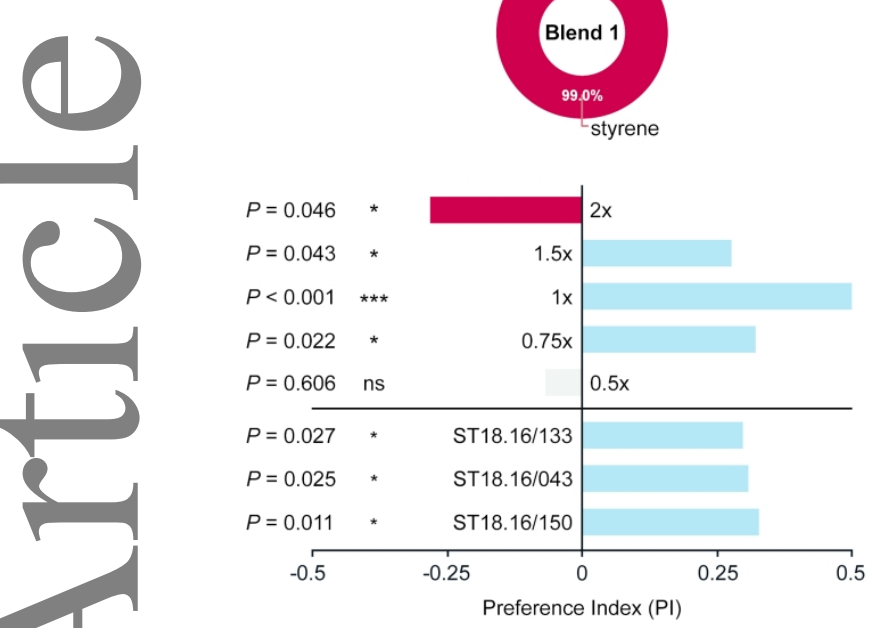

B

1,3-diacetylbenzene

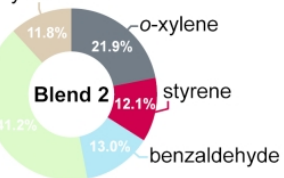

butyl acetate

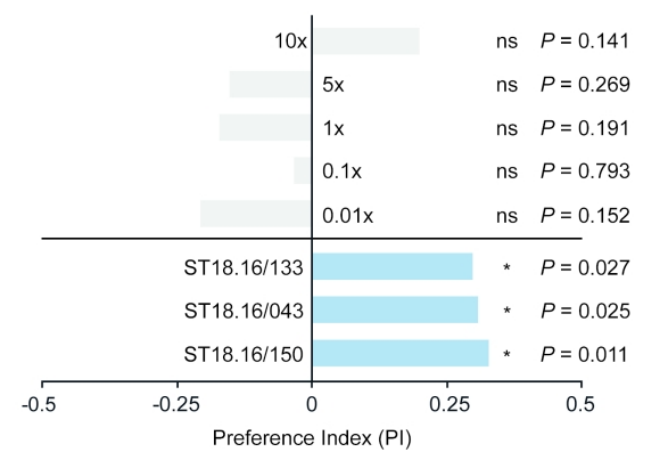

This article is protected by copyright. All rights reserved. 
A
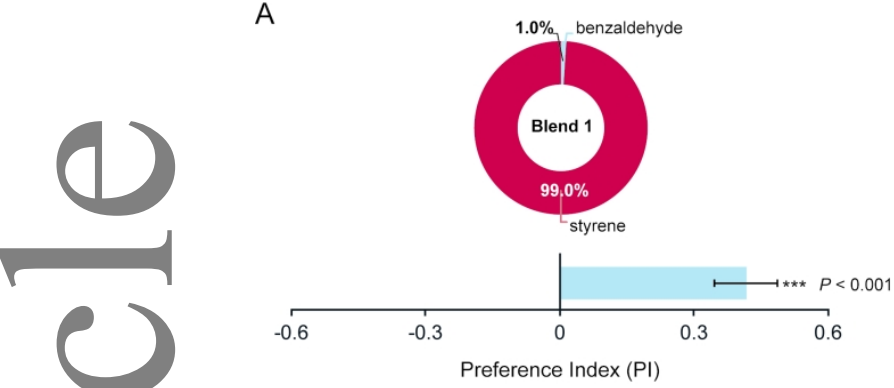

B

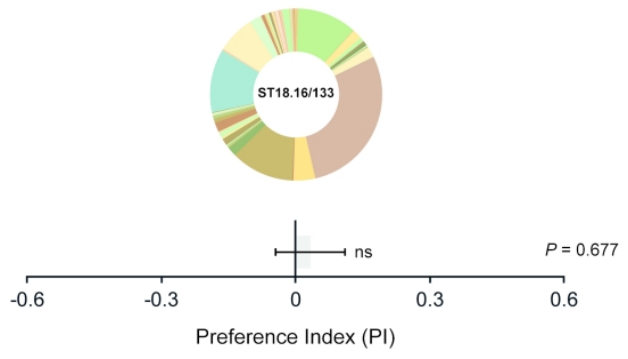

This article is protected by copyright. All rights reserved. 